\title{
Ignatzschineria indica Isolated From a Human Breast Abscess: A Rare Case
}

\author{
Linette Mejias ${ }^{\mathrm{a}}$, Christian Curcio ${ }^{\mathrm{a}}$, Adriana Sanchez ${ }^{\mathrm{a}}$, Bushra Siddique ${ }^{\mathrm{b}}$, Nikolay K. Popnikolov ${ }^{\mathrm{a}}$, \\ Suresh G. Joshic, d, Christopher L. Emery ${ }^{\mathrm{a}, \mathrm{d}}$
}

\begin{abstract}
Ignatzschineria indica is a gram-negative aerobic bacillus, usually isolated from the gut content of adult flesh flies or larvae of Wohlfahrtia magnifica. Ignatzschineria spp. associated with human infection is hypothesized to be related to maggot therapy. We are reporting the first case of Ignatzschineria indica isolated from a breast abscess, along with Proteus penneri and Providencia stuartii in a 76-year-old female. The patient presented with a left breast mass, weight loss, and normal vital signs; physical examination of the left breast showed a fungating mass with foul smelling draining necrotic tissue and a left axillary abscess. A biopsy was performed that revealed an invasive mammary carcinoma (mucinous type) with the tumor ulcerating the overlying skin. Additionally, a culture of the abscess yielded growth of gram-negative rods. The organism was identified as I. indica by $16 \mathrm{~S}$ rRNA gene sequencing. Although the pathogenic capacity of the organism cannot be determined at this point, we are establishing the need for further study of patients with cultures positive for I. indica in order to understand the pathologic implications, and therapy to control this organism in human host.
\end{abstract}

Keywords: Breast abscess; Ignatzschineria; Ignatzschineria india; Myiasis; Sarcophaga

\section{Introduction}

The genus Ignatzschineria is characterized by aerobic, gram-

Manuscript accepted for publication October 17, 2016

aDepartment of Pathology and Laboratory Medicine, Drexel University College of Medicine, Hahnemann University Hospital, Philadelphia, PA, USA

${ }^{b}$ Department of Infectious Diseases, Drexel University College of Medicine, Philadelphia, PA, USA

'Surgical Infection Program, Department of Surgery and Department of Microbiology and Immunology, Drexel University College of Medicine, Philadelphia, PA, USA

${ }^{\mathrm{d} C}$ Corresponding Author: Suresh G. Joshi, Surgical Infection Program, Department of Surgery and Department of Microbiology and Immunology, Drexel University College of Medicine, Mail Stop 435, 245 N. 15th Street, Philadelphia, PA 19102, USA. Email: suresh.joshi@drexelmed.edu; Christopher L. Emery, Department of Pathology and Laboratory Medicine, Hahnemann University Hospital, Philadelphia, PA, USA. Email: christopher.emery@drexelmed.edu

doi: http://dx.doi.org/10.14740/jmc2666w negative, non-spore forming, non-motile and regular rodshaped bacteria, and Ignatzschineria indica was described in 2011 using 16S rRNA gene sequencing by Gupta et al [1]. I. indica has been isolated from the gut content of adult flesh flies, Sarcophaga (Dipteria: Sarcophagidae). The species belonging to Ignatzschineria have not been documented as human pathogens, although related species were isolated from a patient with a maggot-infested wound, including a patient with a diabetic foot ulcer [2]. I. indica has not previously been detected in human sources, especially breast abscess, and we are reporting the first case.

\section{Case Report}

The patient was a 76-year-old African American female with past medical history of hypertension and diabetes. She presented to our hospital with abdominal pain for 1 week with an intensity of eight out of ten (8/10). She denied fever, nausea, vomiting and diarrhea, but complained of constipation of 5 days. Upon physical examination, she was found to have a left side fungating breast mass. The patient stated she did not like hospitals and that was why she did not seek for medical attention regarding the breast mass. Physical examination revealed a left breast fungating mass, replacing the entire breast. The nipple was effaced and there was draining necrotic tissue with a foul smell and a left axillary abscess. The abdomen was soft, non-tender with hypoactive bowel sounds. The rest of the physical exam was unremarkable. Her vitals were temperature of $98.4^{\circ} \mathrm{F}$, blood pressure of 104/60 mm Hg, heart rate of 89 beats per minute (bpm) and a respiratory rate of 14 breaths per minute. A complete blood count $(\mathrm{CBC})$ revealed white blood cell (WBC) count of 15,600 cells $/ \mathrm{mL}$, hemoglobin of $4.8 \mathrm{~g} / \mathrm{dL}$ and a creatinine of $2.07 \mathrm{mg} /$ dL. She was admitted, transfused with two units of packed red blood cells, and was empirically started on vancomycin and piperacillin/tazobactam. She did not experience any febrile episodes. Chest CT scan revealed a lobular left breast mass extending into the left axilla measuring approximately $19 \times 14 \times 16$ $\mathrm{cm}$. Also there were scattered pulmonary nodules with the largest measuring $1.8 \mathrm{~cm}$. A superficial wound swab was taken from the draining breast mass and a biopsy of the left breast mass was performed. The biopsy consisted of two irregular fragments of white tan soft tissue measuring $1.5 \times 1.3 \times 1.0 \mathrm{~cm}$ and $1.0 \times 0.7$ $\times 0.6 \mathrm{~cm}$. The hematoxylin and eosin (H\&E)-stained tissue biopsy revealed an invasive mammary carcinoma (mucinous type) that ulcerated the overlying skin from a tumor size of at least 1.7 

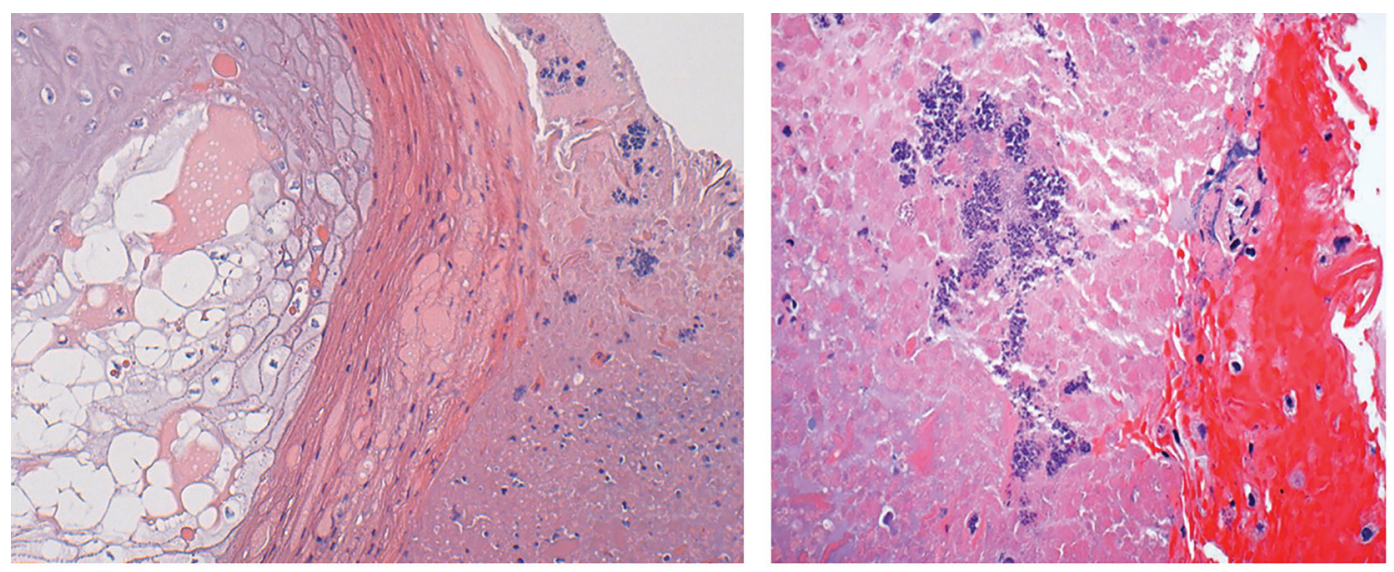

Figure 1. Microphotographs of hematoxylin and eosin (H\&E)-stained tissue biopsy of breast abscess lesion, demonstrating the evidence of necrotic tissues with presence of bacteria (left side, low power; right side, high resolution to demonstrate bacteria).

$\mathrm{cm}$ in greatest dimension with a histological grade of III, and a breast abscess lesion, demonstrating the evidence of necrotic tissues with presence of bacteria shown in Figure 1. The WBC count continued to worsen with an increase of leukocytosis from 15,000 to 19,000 cells $/ \mathrm{mL}$. Renal function improved with hydration and the hemoglobin level improved after blood transfusion. Antibiotics were continued and it was recommended to drain the left breast abscess. On the fifth day of hospitalization, the patient was taken to interventional radiology, and $5 \mathrm{~mL}$ of thick serosanguineous fluid was aspirated from the left breast abscess and sent to the microbiology laboratory. A drain was left in place until the lesion stopped producing any discharge. The patient was treated for 14 days with piperacillin/tazobactam (Zosyn) and the leukocytosis and bandemia decreased. The culture of the abscess showed growth of gram-negative rods, which was presumptively identified by routine biochemical tests following subculturing, and minimum inhibitory concentrations (MIC) were performed by the Vitek2 system. Out of three organisms, two were identified as Proteus penneri and Providencia stuartii. The antimicrobial susceptibility profile is shown in Table 1. The third organism was identified as I. indica by PCR amplification and sequencing of $16 \mathrm{~S}$ rRNA gene, with $100 \%$ match and zero mismatches using the smart gene data base (SmartGene, Lausanne, Switzerland). The susceptibility profile of I. indica is shown in Table 1. Surgeon was consulted who advised that the patient was not a candidate for surgery considering that the left breast mass was very extensive and that she would benefit from chemotherapy prior to a surgery. The patient was discharged and scheduled for follow-up with the hematology/oncology service. She was started on anti-estrogen therapy and her left breast mass decreased significantly, along with the discharge. She was referred for a total mastectomy which she declined.

\section{Discussion}

In our patient, I. indica was isolated in a drain-fluid culture obtained from a breast abscess in the left axillary region. Ignatzschineria is found associated with predominantly the samples from environment and insects such as flesh fly and human infections are rarely reported. Three species were recently reported in association with human infection: I. larvae, I. indica and I. ureiclastica [1]. In 2007, Roudiere et al reported the isolation of Schineria (former name of Ignatzschineria) larvae from the foot of a 39-year-old homeless man with medical history of polyneuropathy related to alcohol abuse [2]. It was found during analysis that the patient had never removed socks and shoes for 2 months, and the wound was contaminated with maggots, the larvae of flies. A Ignatzschineria bacteremia was recently reported from Netherlands [3]. Ignatzschineria spp. along with Providencia stuartii was isolated from the patient and in high abundance from the salivery gland of Lucilia serricata (blowfly) [3, 4]. Baker et al reported for the first time human cases of bacteremia and urinary tract infection from where I. indica was isolated, associated with maggots [5]. In our case breast abscess we did not find maggots or fly larvae, and therefore it is uncertain whether maggot infestation was involved in this infection. Also, the patient was afebrile when the abscess fluid was collected for cultures. The interpretations of the antimicrobial susceptibility testing/MIC results were based on criteria for Enterobacteriaceae, per recommendation of Clinical Laboratory Standards Institute (CLSI) [6]. There are no specific CLSI guidelines for I. indica. Ignatzschineria is resistant to amoxicillin/clavulanic acid. We could not initially identify $I$. indica; and as both Proteus penneri and Providencia stuartii were resistant to ampicillin-sulbactam, we continued empiric therapy with piperacillin/tazobactam. Vancomycin was added for extended coverage. Eventually, the patient responded well to this antimicrobial therapy, but an antimicrobial susceptibility panel has to be standardized for such purpose where monobacterial (Ignatzschineria) infection is identified. Myiasis-producing agents are gaining increasing importance not only due to maggot therapy, and infected wounds but their forensic importance in decomposing human remains [7].

\section{Conclusion}

Despite the isolation and the presence of this bacterium in an 
Table 1. Susceptibility Profiles of Ignatzschineria indica, Proteus penneri and Providencia stuartii

\begin{tabular}{|c|c|c|c|c|c|c|}
\hline \multirow{2}{*}{ Antibiotic } & \multicolumn{2}{|c|}{ P. penneri } & \multicolumn{2}{|c|}{ P. stuartii } & \multicolumn{2}{|c|}{ I. indica } \\
\hline & MIC & Interp. & MIC & Interp. & MIC & Interp. \\
\hline Ampicillin & $\geq 32$ & $\mathrm{R}$ & $\geq 32$ & $\mathrm{R}$ & $\leq 2$ & ND \\
\hline Amplicillin/sulbactam & 8 & $\mathrm{R}$ & ND & $\mathrm{R}$ & ND & ND \\
\hline Amikacin & ND & ND & $\leq 2$ & $\mathrm{~S}$ & $\leq 2$ & $\mathrm{~S}$ \\
\hline Aztreonam & $\leq 1$ & $\mathrm{~S}$ & $\leq 1$ & $\mathrm{~S}$ & $\leq 4$ & $\mathrm{~S}$ \\
\hline Ceftazidime & ND & ND & ND & ND & $\leq 1$ & $\mathrm{~S}$ \\
\hline Ceftriaxone & 2 & I & $\leq 1$ & $\mathrm{~S}$ & $\leq 4$ & ND \\
\hline Cefepime & $\leq 1$ & $\mathrm{~S}$ & $\leq 1$ & $\mathrm{~S}$ & ND & ND \\
\hline Cefoxitin & $\leq 4$ & $\mathrm{~S}$ & $\leq 4$ & $\mathrm{~S}$ & $\leq 8$ & ND \\
\hline Chloramphenicol & ND & ND & ND & ND & $\leq 8$ & ND \\
\hline Ciprofloxacin & ND & ND & ND & ND & $\leq 0.5$ & $\mathrm{~S}$ \\
\hline Ertapenem & $\leq 0.5$ & $\mathrm{~S}$ & $\leq 0.5$ & $\mathrm{~S}$ & $\leq 1$ & ND \\
\hline Gentamicin & $\leq 1$ & $\mathrm{~S}$ & ND & $\mathrm{R}$ & ND & ND \\
\hline Imipenem & 2 & I & 2 & I & $\leq 1$ & $\mathrm{~S}$ \\
\hline Levofloxacin & $\leq 0.12$ & $\mathrm{~S}$ & ND & ND & $\leq 1$ & $\mathrm{~S}$ \\
\hline Meropenem & $\leq 0.25$ & $\mathrm{~S}$ & $\leq 0.25$ & $\mathrm{~S}$ & $\leq 1$ & $\mathrm{~S}$ \\
\hline Piperacillin/tazobactam & $\leq 4$ & $\mathrm{~S}$ & $\leq 4$ & $\mathrm{~S}$ & ND & ND \\
\hline Tetracycline & ND & ND & ND & ND & $\leq 4$ & ND \\
\hline Tigecycline & ND & ND & ND & ND & $\leq 1$ & ND \\
\hline Trimethoprim/sulfamethoxazole & $\leq 20$ & $\mathrm{~S}$ & $\leq 20$ & $\mathrm{~S}$ & $\leq 2 / 38$ & $\mathrm{~S}$ \\
\hline Tobramycin & & & & & $\leq 1$ & $\mathrm{~S}$ \\
\hline
\end{tabular}

MIC: minimum inhibitory concentration $(\mu \mathrm{g} / \mathrm{mL})$; Interp.: interpretation; S: susceptible; R: resistant; ND: no data available.

abscess adjacent to breast cancer, the pathogenic capacity of the organism cannot be definitively determined at this point, since no report describes that this bacterium colonizes or infects humans. There is a need for further study of patients with cultures positive for Ignatzschineria spp., including I. indica, in order to more completely understand the clinicopathologic implications of isolating these bacteria from human specimens.

\section{Conflicts of Interest}

The authors have no financial or other conflicts of interest to declare.

\section{Funding}

None.

\section{Author Contributions}

All authors listed contributed to medical care of the patient and/or contributed to interpretations, and the creation of the case report manuscript.

\section{References}

1. Gupta AK, Dharne MS, Rangrez AY, Verma P, Ghate HV, Rohde M, Patole MS, et al. Ignatzschineria indica sp. nov. and Ignatzschineria ureiclastica sp. nov., isolated from adult flesh flies (Diptera: Sarcophagidae). Int J Syst Evol Microbiol. 2011;61(Pt 6):1360-1369.

2. Roudiere L, Jean-Pierre H, Comte C, Zorgniotti I, Marchandin H, Jumas-Bilak E. Isolation of Schineria sp. from a man. Emerg Infect Dis. 2007;13(4):659-661.

3. Heddema E, Janssen F, van Westreenen H. A case of Ignatzschineria bacteraemia in an unconscious man from the Netherlands. JMM Case Rep. 2016.

4. Singh B, Crippen TL, Zheng L, Fields AT, Yu Z, Ma Q, Wood TK, et al. A metagenomic assessment of the bacteria associated with Lucilia sericata and Lucilia cuprina (Diptera: Calliphoridae). Appl Microbiol Biotechnol. 2015;99(2):869-883.

5. Barker HS, Snyder JW, Hicks AB, Yanoviak SP, Southern P, Dhakal BK, Ghimire GR, et al. First case reports of Ignatzschineria (Schineria) indica associated with myiasis. J Clin Microbiol. 2014;52(12):4432-4434.

6. Clinical Laboratory Standards Institute (CLSI) document M-100 S23, Clinical Laboratory Standards Institute, Wayne, Pennsylvania, 2013. 
7. Sukontason KL, Sanit S, Klong-Klaew T, Tomberlin JK, Sukontason K. Sarcophaga (Liosarcophaga) dux (Dip- tera: Sarcophagidae): A flesh fly species of medical importance. Biol Res. 2014;47:14. 